\title{
The Physiological Functions of Mammalian Endoplasmic Oxidoreductin 1: On Disulfides and More
}

\author{
Thomas Ramming and Christian Appenzeller-Herzog
}

\begin{abstract}
Significance: The oxidative process of disulfide-bond formation is essential for the folding of most secretory and membrane proteins in the endoplasmic reticulum (ER). It is driven by electron relay pathways that transfer two electrons derived from the fusion of two adjacent cysteinyl side chains onto various types of chemical oxidants. The conserved, ER-resident endoplasmic oxidoreductin 1 (Ero1) sulfhydryl oxidases that reduce molecular oxygen to generate an active-site disulfide represent one of these pathways. In mammals, two family members exist, Ero1 $\alpha$ and Ero1 $\beta$. Recent Advances: The two mammalian Ero1 enzymes differ in transcriptional and posttranslational regulation, tissue distribution, and catalytic turnover. A specific protein-protein interaction between either isoform and protein disulfide isomerase (PDI) facilitates the propagation of disulfides from Ero1 via PDI to nascent polypeptides, and inbuilt oxidative shutdown mechanisms in Ero1 $\alpha$ and Ero1 $\beta$ prevent excessive oxidation of PDI. Critical Issues: Besides disulfide-bond generation, Ero1 $\alpha$ also regulates calcium release from the ER and the secretion of disulfide-linked oligomers through its reversible association with the chaperone ERp44. This review explores the functional repertoire and possible redundancy of mammalian Ero1 enzymes. Future Directions: Systematic analyses of different knockout mouse models will be the most promising strategy to shed new light on unique and tissue-specific roles of Ero1 $\alpha$ and Ero1 $\beta$. Moreover, in-depth characterization of the known physical interactions of Ero1 with peroxidases and PDI family members will help broaden our functional and mechanistic understanding of Ero1 enzymes. Antioxid. Redox Signal. 16, 1109-1118.
\end{abstract}

\section{Introduction: Primary Lessons from Yeast}

A COMMON CHARACTERISTIC of a vast majority of secretory and membrane proteins is their need to form disulfide bonds. These covalent linkages, generated between two cysteine side chains by dehydrogenation (oxidation), can either be located within a single polypeptide chain (intramolecular disulfide) or connect two proteins to form dimeric/oligomeric complexes (intermolecular disulfide). Disulfide bonds often play a pivotal role in promoting proper folding of native polypeptide chains entering the endoplasmic reticulum (ER) and in stabilizing the structure of folded proteins destined for the secretory system. An elaborate enzymatic machinery that is responsible for oxidative protein folding, that is, the introduction of disulfide bonds into folding substrates, is present in the ER of all eukaryotic organisms. During this process, disulfide bonds move from one pair of cysteines to another (Fig. 1). These thiol-disulfide exchange reactions are orchestrated by a specialized family of disulfide carrier enzymes, the protein disulfide isomerases (PDIs) (5). Importantly though, the ER is also capable of generating disulfide bonds de novo. While several pathways that can convert diverse types of oxidants into disulfides exist in parallel (13), this reviewalong with a review by Araki and Inaba that elaborately works out the structural and evolutionary point of view (8)will discuss endoplasmic oxidoreductin 1 (Ero1) enzymes, which use molecular oxygen $\left(\mathrm{O}_{2}\right)$ as electron acceptor (and are therefore termed "oxidases").

ERO1 has first been described in baker's yeast as an essential gene $(20,44)$. Its product Ero1p is an ER-resident glycoprotein and a critical determinant for the oxidizing capacity of the yeast cell $(20,44)$. It possesses two redox-active di-cysteine active sites. The "inner active site" is oxidized by a proximally bound flavin adenine dinucleotide (FAD) cofactor $(25,50)$, which itself receives oxidizing equivalents by reducing $\mathrm{O}_{2}$ to hydrogen peroxide $\left(\mathrm{H}_{2} \mathrm{O}_{2}\right)$ (26). The resulting disulfide bond is then transferred from the core of the protein to the "outer active site" (47), which is located in a flexible peptide loop (25). Via its outer active site-also termed the "shuttle disulfide"-Ero1p can directly and specifically oxidize one of the two active-site cysteine pairs in PDI, the archetypal member of the PDI family (21, $22,50)$. This disulfide relay from Ero1p to PDI enables oxidized PDI to subsequently introduce disulfide bonds into folding substrates (Fig. 2) (21, 50).

Division of Molecular and Systems Toxicology, Department of Pharmaceutical Sciences, University of Basel, Basel, Switzerland. 


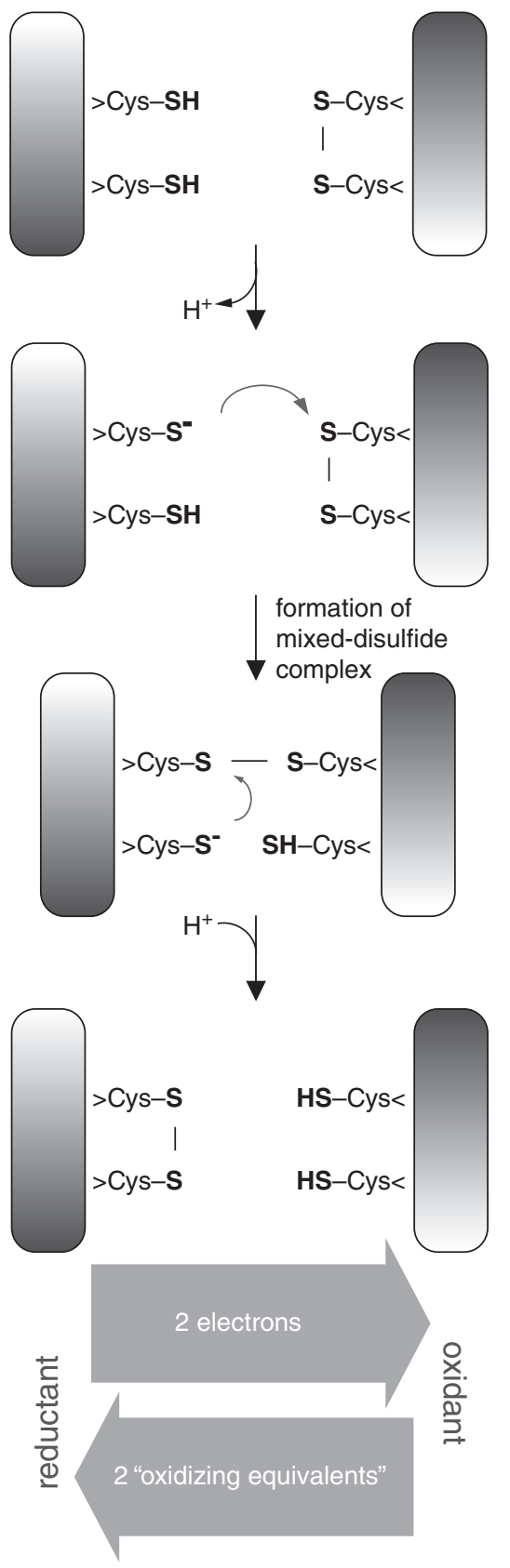

FIG. 1. The thiol-disulfide exchange reaction. Cartoon depicting the mechanism of thiol-disulfide exchange between a reduced (SH) and a disulfide-linked (S-S) cysteine pair (e.g., residing on two separate proteins). Upon deprotonation of one of the cysteinyl thiol groups, the resulting thiolate anion nucleophilically attacks one of the disulfide-bound sulfur atoms, leading to the formation of a mixed-disulfide complex. Deprotonation of and nucleophilic attack by the second thiol group then prompts the formerly reduced pair of cysteines to form a disulfide bond. In the course of this redox reaction, two electrons are transferred from the reductant to the oxidant in exchange of two "oxidizing equivalents."

Since the resolution of mismatched substrate disulfides by reduced PDIs is a fundamental component of oxidative protein folding, an unregulated (hyper-) oxidation of PDI by Ero1p would be undesirable. Thus, a redox-sensitive shutdown mechanism represented by noncatalytic, intra-

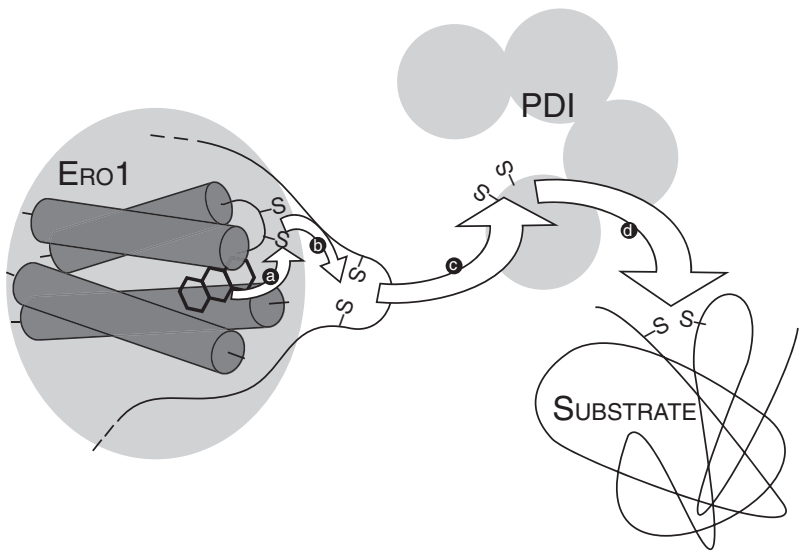

FIG. 2. Disulfide relay from Ero1 via PDI to substrate proteins. A disulfide bond is generated by the transfer of two oxidizing equivalents from the flavin adenine dinucleotide cofactor (shown as three black rings embedded between the four Ero1 core $\alpha$-helices, drawn as dark gray cylinders) to the inner-active-site cysteine pair in Ero1 (step a). After the intramolecular thiol-disulfide exchange reaction (see Fig. 1) between inner and outer active site of Ero1 (step b), the disulfide bond is transferred from the outer active site (located within a flexible loop region in Ero1, depicted by a sinuous black curve) to one of the two di-cysteine active sites in PDI (step c). As a consequence, PDI is capable of introducing disulfide bonds into nascent polypeptides (dubbed "substrate") through thiol-disulfide exchange (step d). Arrows denote the flow of two oxidizing equivalents; cysteine pairs are depicted only by their sulfur atoms (S); the four thioredoxin-like domains in PDI are represented by gray circles. Ero1, endoplasmic oxidoreductin 1; PDI, protein disulfide isomerase.

molecular disulfide bonds effectively impairs Ero1p activity $(27,48)$. As the oxidation state of these cysteine pairs is controlled by the ER redox poise, a regulatory feedback loop ensues in which Ero1p is solely active when new disulfides are required (48). Taken together, many of the conserved features of Ero1 sulfhydryl oxidases including their fold, mechanism of action, physiological relevance, substrate specificity, and the principle of their tunable activation status have been unraveled in experiments with the yeast enzyme.

\section{What Yeast Has Failed to Teach Us}

Orthologs of Ero1p exist in all eukaryotes (8). Mammalian genomes harbor two Ero1-like genes, ERO1L and ERO1LB, which encode Ero1 $\alpha$ and Ero1 $\beta$, respectively $(14,42)$, and will be the subject of this review. Interestingly, the study of Ero1 $\alpha$ and $\operatorname{Ero} 1 \beta$ has not only unveiled many parallels to the yeast enzyme, but also a number of important differences. Most prominent among these, the mammalian Ero1 genes appear to be nonessential, as evidenced by the viability of mice carrying mutated copies of both ERO1L and ERO1LB (55). The mutated genes feature an intronic "gene trapping" insertion containing a strong viral splice acceptor. As a consequence, the vast majority of mRNAs derived from the ERO1L/ERO1LB loci will give rise to nonfunctional, truncated proteins.

Lipopolysaccharide-activated spleen cells (LPS blasts) isolated from the mutant mice are nearly indistinguishable from their wild-type counterparts with regard to the efficiency of 
oxidative folding of immunoglobulin $\mathrm{M}(\operatorname{IgM})(55,56)$. With one known exception (the oxidative folding of proinsulin in the $\beta$-cells of the pancreas, discussed further below), it is therefore reasonable to assume that the overall pace of disulfide-bond formation is not severely compromised in ERO1L/ERO1LB mutant mice and does not phenocopy the fatal situation in Ero1p-deficient yeast cells.

These observations can be explained by alternative mechanisms for de novo disulfide-bond generation in the mammalian ER (13) and/or by incomplete gene trapping, which would allow the low-level synthesis of operational Ero1 molecules. Indeed, residual amounts of $\operatorname{Ero1} \beta$ were detected on mRNA level in cardiomyocytes (16) and on protein level in pancreatic tissue and LPS blasts from Ero1 double mutant mice (55). In all cell types studied so far, only a minor portion of Ero1 $\alpha$ and, presumably, Ero1 $\beta$ is maintained in an active form $(6,11,16)$ (see also below). Consequently, when shifted to increased activity through redox regulation, the residual levels of Ero1 $\beta$ in ERO1L/ERO1LB compound mutant mice might actually suffice to support disulfide-bond formation. Along the same lines, yeast cells can proliferate in the presence of very low amounts of Ero1p. Sufficient levels of Ero1p to allow for growth of an ero1-null strain can be provided by a plasmid encoding ERO1 under the control of a galactoseinducible promoter even when cells are grown in glucose (where the promoter is largely repressed) (Carolyn S. Sevier, pers. comm.). It is therefore important to consider that the designation of ERO1L/ERO1LB mutant mice as "knockout animals" can provoke an underestimation of the functional significance of Ero1 enzymes in mammals. Interestingly, the high proliferation rate of immortalized mouse embryonic fibroblasts is profoundly affected by the ERO1L/ERO1LB compound mutation (Ester Zito, David Ron, and Christian
Appenzeller-Herzog, unpublished observations), suggesting that under in vitro conditions, normal levels of Ero1 enzymes are required for optimal cell growth. On the other hand, flies homozygous for a nonsense allele of their single ERO1L gene develop almost normally (49), which strongly suggests the existence of ERO1L-independent pathways for disulfide-bond formation.

In addition, while both Ero1p and Ero1 $\alpha$ (and, most likely, Ero1 $\beta$ ) are soluble ER proteins, which are peripherally membrane-associated $(14,20,42)$, only Ero1p possesses a C-terminal membrane-targeting domain (43). Further differences between Ero1p and the mammalian Ero1 enzymes include a distinct set of regulatory disulfide bonds for the shutdown of oxidase activity and different mechanisms of substrate recognition and oxidation. These issues will be covered in subsequent sections.

\section{Ero1 Enzymes Are Feedback-Regulated Sulfhydryl Oxidases}

Of note, not all of the cysteines within the primary sequence of Ero1 are positionally conserved from yeast to mammals. While the inner and outer active-site cysteines (Cys ${ }^{394}-\mathrm{Cys}^{397}$ and $\mathrm{Cys}^{94}$-Cys ${ }^{99}$ in human Ero1 $\alpha$ ) and the long-range disulfide connecting the two active-site peptides $\left(\mathrm{Cys}^{85}-\mathrm{Cys}^{391}\right.$ in Ero1 $\alpha$ ) are preserved, intramolecular disulfide bonds homologous to the regulatory $\mathrm{Cys}^{143}-\mathrm{Cys}^{166}$ and $\mathrm{Cys}^{150}-\mathrm{Cys}^{295}$ in Ero1p are absent from both Ero1 $\alpha$ and Ero1 $\beta$ (Fig. 3). Amino acid substitution and mass spectrometry analysis revealed that Ero $1 \alpha$ in its most oxidized redox state forms a disulfide bond between Cys ${ }^{94}$ and Cys ${ }^{131}(6,10)$. As Cys ${ }^{94}$ is a constituent of the outer active site, $\mathrm{Cys}{ }^{94}-\mathrm{Cys}{ }^{131}$ has to be resolved to allow disulfide shuttling to PDI. Interestingly, also Cys ${ }^{99}$
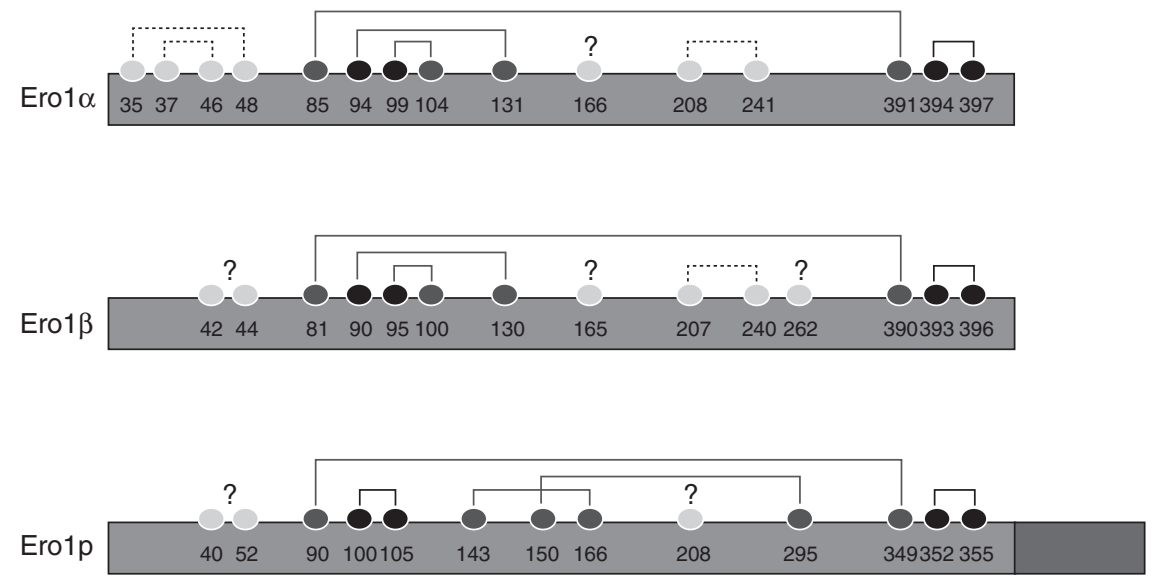

FIG. 3. Cysteine connectivity of oxidatively silenced Ero1 enzymes. Ero1 $\alpha$, Ero1 $\beta$, and Ero1p polypeptides are depicted by gray bars. Numbered dots represent the positions of cysteine residues within the respective Ero1 sequences, and brackets connecting two dots show intramolecular disulfide bonds. Ero1 $\alpha$, Ero1 $\beta$, and Ero1p possess two di-cysteine active sites (black dots), the C-terminal inner active sites $\left(\mathrm{Cys}^{394+397}, \mathrm{Cys}^{393+396}, \mathrm{Cys}^{352+355}\right.$ ) and the N-terminal outer active sites $\left(\mathrm{Cys}^{94+99}\right.$, $\mathrm{Cys}^{90+95}$, Cys $\left.{ }^{100+105}\right)$. Regulatory disulfides, which negatively control oxidase activity, are pictured by dark gray brackets. Not all of the regulatory cysteines (dark gray) are positionally conserved between Ero1p and Ero1 $\alpha / \beta$, and disulfide linkages between regulatory and outer-active-site cysteines only exist in the mammalian enzymes. Note that the functional coloring of the long-range disulfide $\left(\mathrm{Cys}^{85}-\mathrm{Cys}^{391}, \mathrm{Cys}^{81}-\mathrm{Cys}^{390}, \mathrm{Cys}^{90}-\mathrm{Cys}^{349}\right.$ ) and the cysteine connectivity of Ero1 $\beta$ is speculative at present (see main text for details). Cysteines of structural or unknown function (light gray) are connected with dotted brackets in case of disulfide-bond connection. Question marks denote that no conclusive redox state has been demonstrated, yet. Ero1p possesses an additional C-terminal domain that is responsible for its tethering to the membrane (dark gray box). For more detailed information regarding redox-state-dependent changes in the cysteine connectivity refer to the main text. 
forms a "nonactive-site" disulfide with $\mathrm{Cys}^{104}$ in the shut-off state of Ero1 $\alpha$ (10). Like Cys ${ }^{131}$, Cys $^{104}$ has no equivalent in Erolp.

The identification of these new types of regulatory disulfide, which are likely to be present in $\operatorname{Ero1} \beta$, too (53), has implications for the redox-driven mechanisms of enzyme (in)activation. Thus, in an oxidizing ER environment when reduced substrates for Ero1 $\alpha$ are scarce, newly produced disulfide bonds arising from the inner active site will be "stored" as Cys ${ }^{94}$-Cys ${ }^{131}$ and Cys ${ }^{99}$-Cys ${ }^{104}$. Molecularly, a likely scenario would be the nucleophilic attack of the shuttle disulfide by $\mathrm{Cys}^{131}$ (giving rise to Cys ${ }^{94}$-Cys ${ }^{131}$ ) followed by the transfer of a second disulfide from the inner active site via the transient formation of $\mathrm{Cys}^{99}{ }^{9} \mathrm{Cys}^{394}$ [in analogy to Ero1p; (47)], which is then resolved through nucleophilic attack by $\mathrm{Cys}^{104}$. As to the reactivation under reducing conditions, the situation is less straightforward. Two electrons are required to break either of the two regulatory disulfide bonds, before the shuttle disulfide can be reformed through nucleophilic attack. The finding that the concentration of reduced PDI in the ER influences the extent of $\mathrm{Cys}^{94}$-Cys ${ }^{131}$ formation suggests PDI as the reductant (6). However, PDI has proven to be an ineffective activator of Ero1 $\alpha$ in a reconstituted reaction $(9,10,15$, 52). In keeping with the predominantly inactive state of Ero1 $\alpha$ in the ER $(6,11,16)$, this relative resistance of Ero1 $\alpha$ toward PDI-mediated reduction could be a critical determinant of ER redox control rather than a manifestation of its poor catalytic proficiency. Indeed, when the thiol load of the ER is maximized by treatment with a strong reducing agent, the Ero $1 \alpha-$ dependent re-formation of disulfides is exceptionally fast upon washout of the reductant (7). These findings are consistent with the concept that Ero1 $\alpha$ is an environmentdependent sulfhydryl oxidase, the activity of which is governed by the redox state of its own substrate(s).

In contrast to the aforementioned disulfide bonds, the longrange disulfide, which is conserved in all Ero1 orthologs, does not involve any active-site cysteines (Fig. 3). Two alternative views exist on the role of this disulfide during catalysis. Based on the finding that purified Ero1 $\alpha$ C85A-C104A-C131AC391A - although well-folded-is less active in an oxidase assay than Ero1 $\alpha$ C104A-C131A (9), one opinion holds that $\mathrm{Cys}^{85}$-Cys ${ }^{391}$ must be intact for efficient substrate oxidation. The second view, which we tend to favor, suggests a rearrangement of this disulfide during enzyme activation. Thus, an as yet unidentified cysteinyl thiolate anion might attack, for example, $\mathrm{Cys}^{85}$ and thereby free $\mathrm{Cys}^{391}$ to create a new short-range disulfide in Ero1 $\alpha$ that facilitates the communication between inner and outer active site. It can also be speculated that the presumed isomerization reaction is not readily triggered through intramolecular attack, but instead catalyzed by a thiol-disulfide isomerase such as ERp44 or another PDI that could initially resolve the long-range disulfide (for discussion of mixed-disulfide interactions of Ero1, see below). In potential support of this second view, activated Ero1 $\alpha$ (10) and Ero1 $\beta$ (53) virtually co-migrate with the fully reduced forms on nonreducing gels. In addition, presumably catalytic mixed-disulfide complexes between Ero1 $\alpha$ or Ero1 $\beta$ and PDI trapped in living cells after treatment with a reductant display markedly decreased gel mobility-indicative of long-range disulfide resolution-as compared with the complexes trapped at steady state (7). Intriguingly, the crystal structure of a hyperactive Ero $1 \alpha$ C104A-C131A mutant, which still harbors $\mathrm{Cys}^{85}{ }^{-\mathrm{Cys}^{391}}{ }^{3}$, does not reveal any obvious pathway for $\mathrm{O}_{2}$ to reach the protein-embedded FAD moiety (29). We speculate that structural flexibility upon disruption of $\mathrm{Cys}^{85}$-Cys ${ }^{391}$ will be instrumental for the emergence of such an aqueous $\mathrm{O}_{2}$ channel. A detailed study on the reductive activation of Ero1p has also indicated that a subfraction of the long-range disulfide is resolved prior to the catalysis of substrate oxidation (27).

\section{Mechanisms of selection of specific sulfhydryl substrates}

Among the total of $\sim 20$ PDI-like proteins in humans (5), PDI itself has been shown to be the major substrate for Ero1mediated oxidation. Numerous cues in favor of this view exist including data from both cell culture and in vitro assays (6, 7, $29,53)$. Ero1 $\alpha$ recognizes PDI by a lock-and-key principle irrespective of whether or not the redox-active cysteines are present in PDI $(29,41)$. Thus, initial binding prior to the formation of a catalytic mixed disulfide occurs through noncovalent interactions. It has been conclusively demonstrated that these interactions are of hydrophobic nature (29). They involve a protruding $\beta$-hairpin in Erol $\alpha$, which contains a critical tryptophan residue at its very tip $\left(\operatorname{Tr} p^{272}\right.$ ), and a hydrophobic cleft in the substrate-binding domain of PDI (36). Even though experimental data were exclusively generated with Ero $1 \alpha$, both the hairpin structure and the crucial tryptophan are present in Ero1 $\beta$ as well (Fig. 4). Accordingly, the principle of substrate recognition is probably conserved among the mammalian isoforms. In addition, as hinted by in silico complex modeling (36), this mode of interaction presumably facilitates the specific thiol-disulfide exchange between the $\mathrm{C}$ terminal active-site domain of PDI and the shuttle disulfide in Ero1 $\alpha(7,9,10,15,52)$. In contrast, Ero1p harbors no tryptophan-containing $\beta$-hairpin (25) and preferentially oxidizes the N-terminal active-site domain of yeast PDI (51).

As opposed to the bona fide substrate PDI, its homolog ERp44 can efficiently bind to Ero $1 \alpha$ even in the absence of the $\beta$-hairpin (36). Further, equal amounts of ERp44-Ero1 $\alpha$ mixed disulfides are detected with all single-cysteine mutants of Ero1 $\alpha$ (12), indicating that ERp44 can attack at least one disulfide other than the shuttle disulfide with its active-site cysteine. Besides these, also other PDI-family members-although inferior substrates for oxidation $(29,53)$ - form mixed disulfides with Ero1 $\alpha$ and Ero1 $\beta$ within cells under steady-state conditions $(7,30,46)$. As pointed out for PDI (7), these complexes most likely involve an oxidized (noncatalytic) form of Ero1 $\alpha$. In addition, they are not strictly dependent on the presence of an intact shuttle disulfide $(7,12)$ so that they might be formed in analogy to the ERp44Ero1 $\alpha$ interaction. The physiological roles of these covalent complexes are currently unclear.

\section{Ero1 $\alpha$ and Ero1 $\beta$ : Functional substitutes or brothers in arms?}

While the above sections have highlighted many shared catalytic characteristics between Ero1 $\alpha$ and $\operatorname{Ero} 1 \beta$, the two homologs have also diverged in a number of features. For instance, the transcriptional regulation of ERO1L and $E R O 1 L B$ is different, which undoubtedly contributes to the distinct expression profiles in human tissues (42) (Fig. 5). The high Ero1 $\beta$ levels in insulin-producing $\beta$-cells of the pancreas are maintained by the key pancreatic transcription factor 


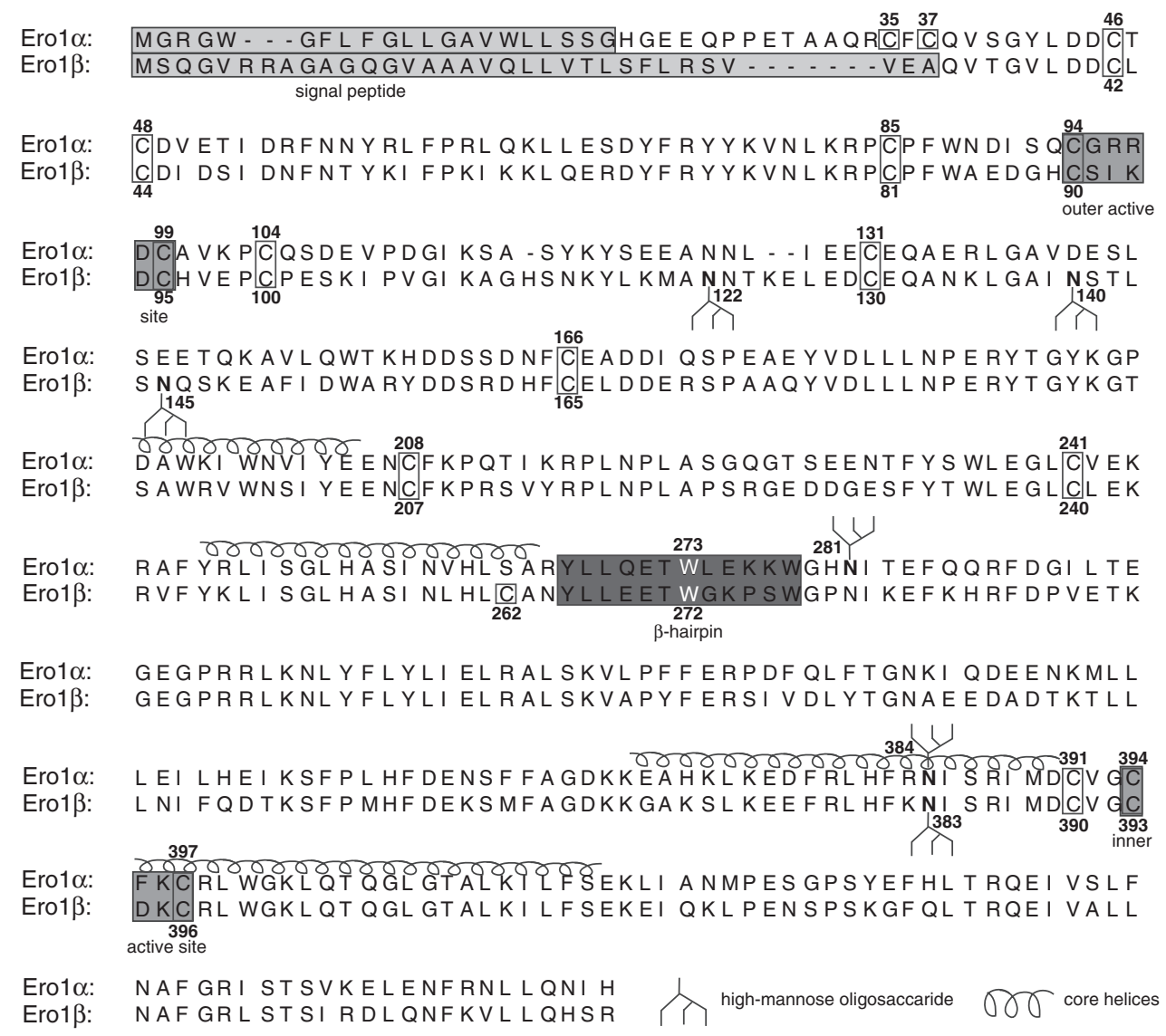

FIG. 4. Annotated sequence alignment of human Ero1 $\alpha$ and Ero1ß. The aligned amino acid sequences are shown in single letter code. Both proteins possess an N-terminal ER-targeting signal peptide (light gray boxes; predicted by the SignalP 3.0 program available at www.cbs.dtu.dk/services/SignalP), an inner and outer di-cysteine active site (gray boxes), four core $\alpha-$ helices (marked by coiled hairlines above the sequence) and a tryptophan (white)-containing $\beta$-hairpin (dark gray box) that is crucial for the interaction with PDI. Cysteine residues are highlighted by black-framed boxes, and asparagine residues within $\mathrm{N}$-glycosylation consensus sites by bold letters and the attachment of a schematized high-mannose oligosaccharide. Numbers next to cysteines, N-glycosylation sites, and the PDI-binding tryptophan show the respective position in the amino acid sequence. ER, endoplasmic reticulum.

PDX1 (31). The importance of Ero1 $\beta$ in these cells is highlighted by the finding that the oxidative maturation of proinsulin is delayed in pancreatic islets from ERO1LB mutant mice, which manifests in a diabetic phenotype (55). Intriguingly, this phenotype is neither complemented by a compensatory increase in Ero1 $\alpha$ levels nor exacerbated by additional mutation of ERO1L, which argues against redundancy of Ero1 isoforms in the endocrine pancreas of mice (55). $E R O 1 L B$ is also a target of the ER-stress-responsive transcription factor ATF6 $\alpha$ (1) that is preferentially activated under reducing ER conditions (39).

$E R O 1 L$, on the other hand, is a transcriptional target of HIF $1 \alpha$ that is upregulated in response to hypoxia or hypoglycemia $(23,37)$. Indeed, Ero1 $\alpha$ is instrumental in counteracting ER hypo-oxidation brought about by hypoxic treatment of cells (16). In addition, the expression of ERO1L is enhanced during adipogenesis, which depends on the nuclear hormone receptor peroxisome proliferator-activated receptor gamma (PPAR $\gamma)$ (45). Finally, Ero1 $\alpha$ is induced during a late stage of ER stress signaling through the binding of C/EBP homologous protein (CHOP; also known as Gadd153) to its promotor (34) (see below).
Ectopic expression of Ero1 $\beta$-in contrast to Ero1 $\alpha$ that has no discernible effect-moderately increases the oxidation of ER oxidoreductases and glutathione (6). This is likely owing to the relative lability of the regulatory disulfide bonds in Ero1 $\beta$, since mutation of $\mathrm{Cys}^{100}$ and $\mathrm{Cys}^{130}$ in Ero1 $\beta$ does not activate the purified enzyme to the same extent as the equivalent mutations in Ero1 $\alpha$ (53). Further, wild-type Ero1 $\beta$ shows higher rates of oxygen consumption during in vitro substrate oxidation compared with Ero1 $\alpha$ (53). At the same time, however, the initial slope of glutathione re-oxidation after complete chemical reduction is less pronounced in Ero $1 \beta$ - than in Ero1 $\alpha$-overexpressing cells (7). Thus, the catalytic turnover rates of Ero1 $\alpha$ and $\operatorname{Ero} 1 \beta$ differ in a context-dependent manner.

Can these disparities be explained at the molecular level? One of the two intercalating amino acids between the inner active-site cysteines in Ero1 $\alpha$ and Ero1 $\beta$ is different (Fig. 4). When mutated to the $\operatorname{Ero} 1 \beta$ active-site sequence, the catalytic turnover number of Ero1 $\alpha$ increases (53). Moreover, Ero1 $\beta$ harbors an additional cysteine at position 262 (Fig. 4), which has been proposed to form a unique type of regulatory disulfide together with $\mathrm{Cys}^{100}$ (53). However, as predictable by homology to Ero1 $\alpha, \mathrm{Cys}^{262}$ is located at the end of one of the 


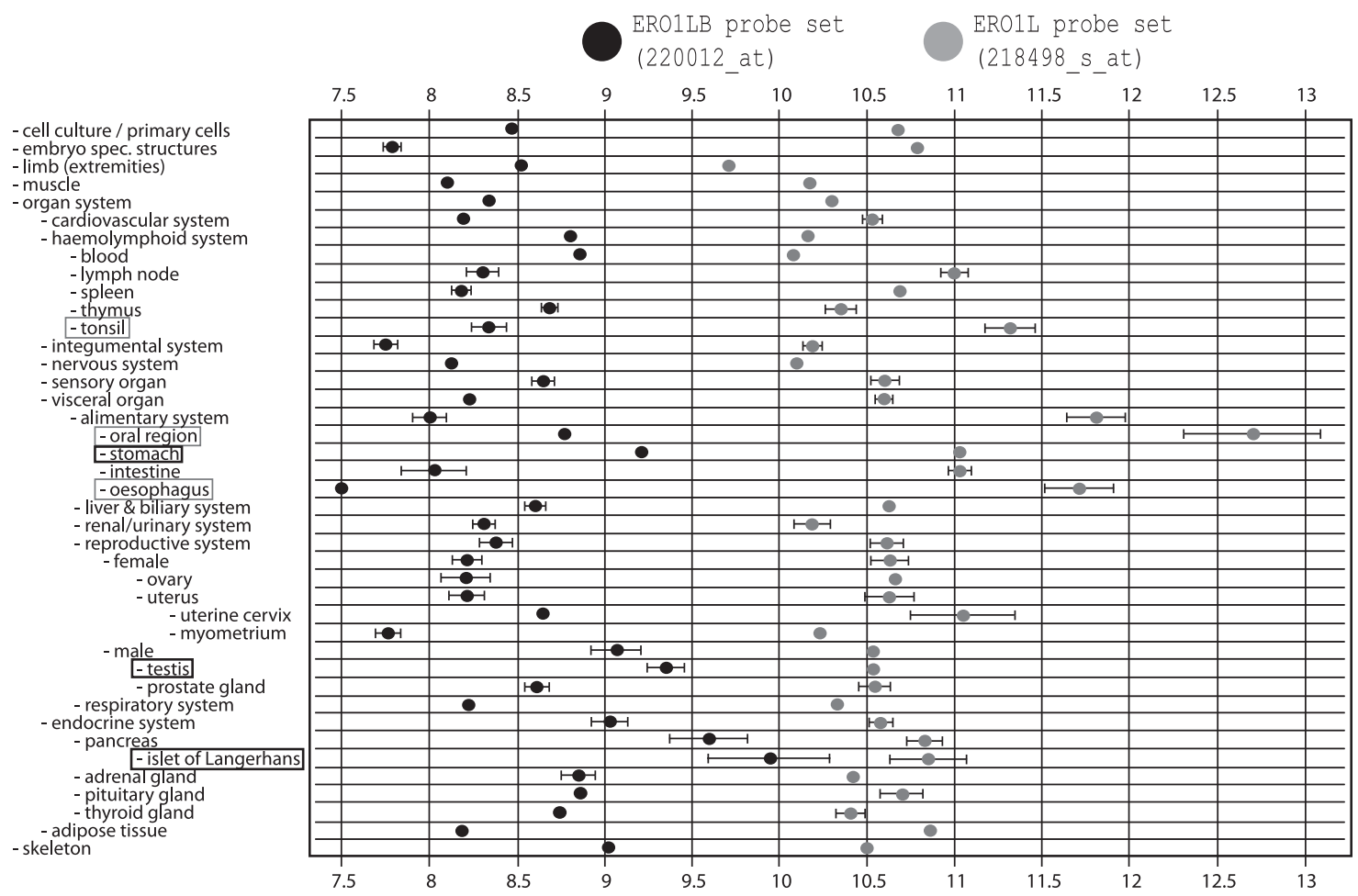

FIG. 5. Tissue-specific mRNA levels of human ERO1L and ERO1LB. Expression levels of ERO1L and ERO1LB mRNAs (encoding Ero1 $\alpha$ and Ero1 $\beta$, respectively) in a range of human tissues shown as signal intensity on Affymetrix Human Genome U133A array (probe sets: 218498_s_at for ERO1L and 220012_at for ERO1LB). The figure was generated with all available datasets using the Genevestigator software (www.genevestigator.com/gv). The signal intensity on the abscissa is expressed on an arbitrary, logarithmic scale. The abundance of ERO1L mRNA is overall higher than that of ERO1LB. ERO1LB expression peaks in the stomach, the testis, and the pancreatic islets of Langerhans (highlighted with dark gray boxes). In contrast, elevated levels of ERO1L mRNA can be detected especially in tonsils, oral region, and oesophagus (highlighted with light gray boxes).

four core $\alpha$-helices and immediately upstream of the PDIinteracting $\beta$-hairpin loop (Fig. 4). We therefore consider it unlikely that this region adopts a fundamentally different conformation in Ero1 $\beta$. In a structural homology model, the side chain of $\mathrm{Cys}^{262}$ is located $\sim 30 \AA$ away from $\mathrm{Cys}^{100}$ and buried in the structure (data not shown). Finally, Ero1 $\beta$ harbors three unique $\mathrm{N}$-glycosylation sites in the peptide surrounding $\mathrm{Cys}^{130}$ (Fig. 4). In the model, the glycosylated asparagines are solvent exposed and positioned between the PDI-binding site and the shuttle disulfide (data not shown), raising the possibility that substrate recruitment might be modulated by the presence of bulky oligosaccharides. Overall, Ero1 $\beta$-basally expressed at low levels (Fig. 5) and an early target of the ER stress response (1) - represents an effective stress oxidase that can counteract ER hypo-oxidation. Ero1 $\alpha$, on the other hand, likely fulfills a tightly regulated housekeeping function with regard to disulfide generation. Whether and to what extent the two isoforms functionally and/or physically interact in tissues where they are coexpressed remains to be examined.

\section{Ero1 $\alpha$ Is Critical for ER-Stress-Induced Apoptosis}

Besides the regulated production of disulfides, Ero1 $\alpha$ has additional roles. A major fraction of Ero1 $\alpha$ molecules localizes to so-called mitochondria-associated membranes (MAMs), a subdomain of the ER that is tethered to mitochondria $(3,24)$. When activated by exogenous reductants or under hypoxic conditions, Ero1 $\alpha$ relocates to the bulk of the ER (24), suggesting that its function at the MAM might be independent of its activity as an oxidase. The excitable calcium channels of the inositol 1,4,5-trisphosphate receptor $\left(\mathrm{IP}_{3} \mathrm{R}\right)$ family are also enriched in MAMs, and $\mathrm{IP}_{3} \mathrm{R}$-facilitated calcium shuttling to cytosol and mitochondria is a critical branch of apoptotic cell death signaling during severe ER stress (17). One of the switches that positively regulate such calcium flow is Ero1 $\alpha$ (32), which is induced by the ER stress-dependent, apoptogenic transcription factor CHOP (34). Since the channeling activity of $\mathrm{IP}_{3} \mathrm{R}$ subtype 1 is impeded by reversible binding of ERp44 (28), elevated levels of Ero1 $\alpha$ - a preferred ligand of ERp44 $(2,36)$ - likely activate $\mathrm{IP}_{3} \mathrm{R} 1$ during ER stress by sequestering ERp44 (in analogy to the mechanism described in the next section). Conversely and similar to the knockdown of $\mathrm{IP}_{3} \mathrm{R} 1$, silencing of Ero1 $\alpha$ in macrophages inhibits ER-stressinduced apoptosis by lowering ER calcium release (32). Recently, knockdown of Ero1 $\alpha$ in HeLa cells has been demonstrated to predominantly hamper the accumulation of calcium in mitochondria upon stimulation of $\mathrm{IP}_{3} \mathrm{Rs}$ (and only marginally in the cytosol), which is in agreement with the enrichment of Ero1 $\alpha$ in MAMs (3).

An alternative, although less plausible possibility is that Ero $1 \alpha$ does not enhance $\mathrm{IP}_{3} \mathrm{R} 1$ activity by lowering the availability of ERp44 but by hyperoxidizing the ER. The inhibitory association with ERp44 depends on two reduced cysteinyl thiols in $\mathrm{IP}_{3} \mathrm{R} 1$ (28), the oxidation of which upon ER stress could consequently lead to channel activation. In 
potential support of this, antioxidant treatment mimicked the inhibitory effect of Ero1 $\alpha$ knockdown on ER calcium release (32). However, as the CHOP-Ero $1 \alpha-\mathrm{IP}_{3} \mathrm{R} 1$ signaling pathway induces NADPH oxidase 2-derived reactive oxygen species (ROS), which in turn further amplify CHOP (33), the application of antioxidants did not necessarily exert its effect by counteracting Ero1 $\alpha$ activity. Moreover, given the tight regulatory mechanisms that prevent excessive disulfide and $\mathrm{H}_{2} \mathrm{O}_{2}$ synthesis by Ero1 $\alpha$ (see above), its increased expression does not per se hyperoxidize the ER $(6,7)$. The probably minor contribution of Ero1-derived ROS to ER-stressinduced cell death has recently been evaluated in detail elsewhere (4).

\section{Role of Ero1 $\alpha$ in ERp44-Mediated ER Retention/Retrieval}

Apart from their involvement in calcium signaling, Ero1 $\alpha$ and ERp44 orchestrate the secretion of diverse disulfidelinked dimers and higher order oligomers by the reversible formation of mixed-disulfide ERp44-protein adducts (Fig. 6).
ERp44 binds to substrates via its nonclassical CXXS active-site motif (2), which lacks a resolving cysteine and therefore renders these intermolecular disulfide adducts longer-lived than typical catalytic complexes. Moreover, despite its C-terminal ER retrieval signal ERp44 is atypically enriched in the ERGolgi intermediate compartment (ERGIC)/cis-Golgi $(35,54)$, which favors a model in which ERp44 retrieves substrates from ERGIC/cis-Golgi to ER.

What is the role of Ero1 $\alpha$ in the intracellular retention of secretory proteins? Increased expression of Ero1 $\alpha$ displaces ERp44 from most of its endogenous substrates (2), indicating it to be a preferred mixed-disulfide ligand of ERp44. Accordingly, the secretion of ERp44 retention substrates such as IgM (2), the adipose-derived hormone adiponectin (54), or sulfatase modifying factor 1 (SUMF1; also known as formylglycine-generating enzyme) $(19,35)$ is enhanced upon overexpression of Ero1 $\alpha$ (Fig. 6). In the case of adipocytes, endogenous induction of Ero $1 \alpha$ by PPAR $\gamma$ agonists is physiologically relevant during differentiation and stimulation (45, 54). Although speculative, vascular endothelial growth factor

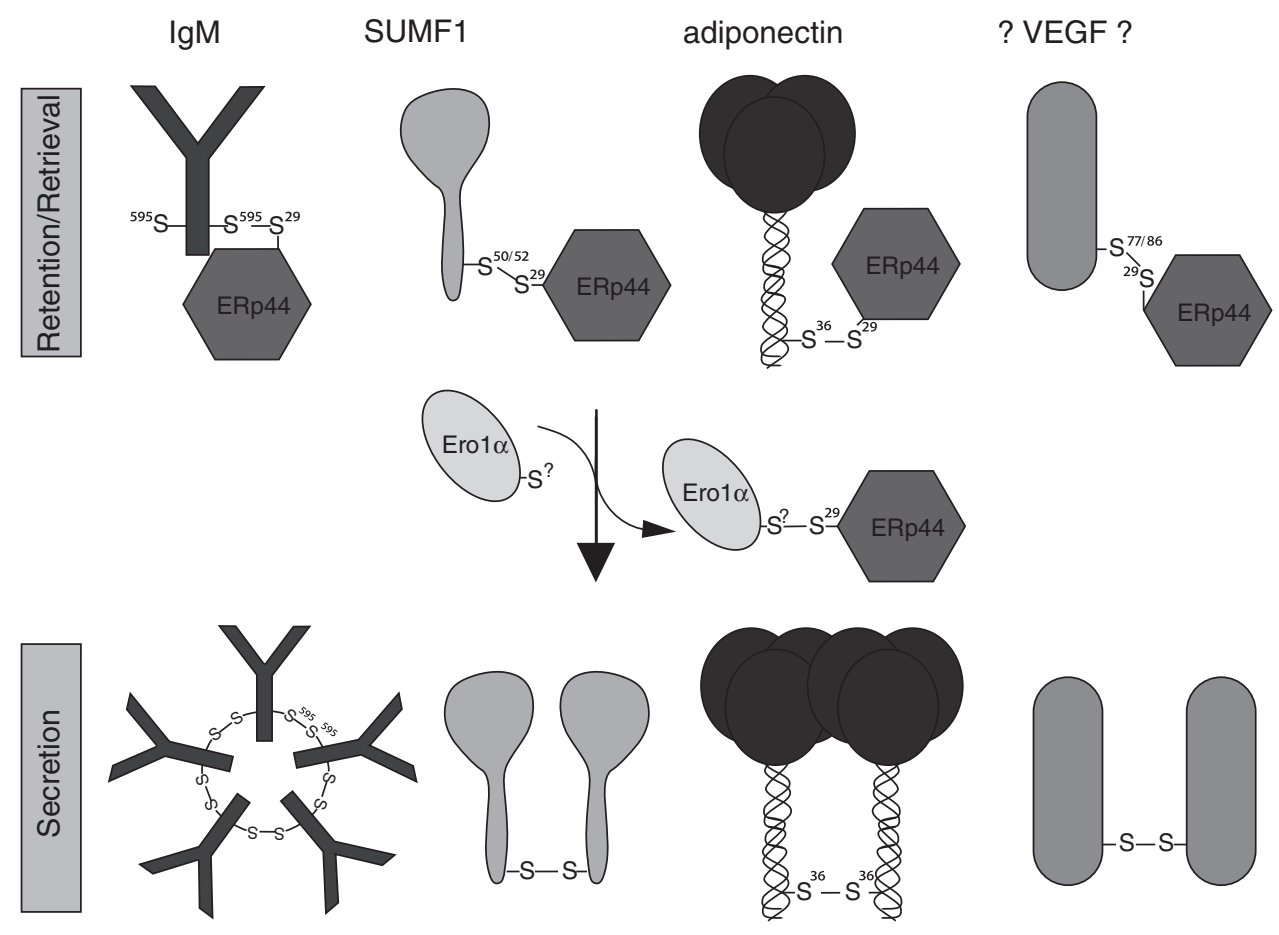

FIG. 6. Interplay between Ero1 $\alpha$ and ERp44 governs the secretion of various disulfide-linked dimers/oligomers. ERp44 mediates ER retention or retrieval from ERGIC/cis-Golgi of various immature monomers destined for dimerization/oligomerization and secretion. In this process, substrate binding by ERp44 is stabilized by formation of an interchain disulfide bond involving $\mathrm{Cys}^{29}$ in ERp44. Upon exogenous overexpression or endogenous induction, Ero1 $\alpha$ can-most likely by a competitive mechanism-displace these substrates from ERp44, thus promoting their maturation into disulfide-linked protein complexes and subsequent secretion. In addition to the known ERp44 substrates IgM, SUMF1, and adiponectin, VEGF displays similar properties, rendering it a potential ERp44 substrate (as indicated by the question marks). While the cysteine residues responsible for ERp44-association and oligomerization have been identified in the substrate proteins, it is still unclear, whether a specific cysteine in Ero1 $\alpha$ is involved or Ero1 $\alpha$ acts as a multivalent mixed-disulfide partner of ERp44 (question mark). The graphical depiction of the structure of the mature IgM pentamer (that contains two heavy and two light chains per monomer) is simplified, and the existence of alternative oligomers (involving a J-chain) is neglected. The Nterminal domains of the adiponectin trimer form a collagen-like triple helix that can dimerize (or multimerize, not shown) through $\mathrm{Cys}^{36}$-mediated interchain disulfide-bond formation. Regarding SUMF1, it is important to point out that the drawn mechanism of Ero1 $\alpha$-induced secretion only applies to overexpressed protein (endogenous SUMF1 is an ER-resident enzyme). Cysteine residues are represented only by their sulfur atoms, S; numbers, where indicated, show the position of the cysteine in the human amino acid sequence. ERGIC, ER-Golgi intermediate compartment; IgM, immunoglobulin M; SUMF1, sulfatase modifying factor 1; VEGF, vascular endothelial growth factor. 
(VEGF) might also add to the list of ERp44 substrates, as its secretion, which is prominent in hypoxic tumors, is positively regulated by Ero1 $\alpha$ (37) (Fig. 6).

All secretory ERp44 substrates identified so far (and VEGF) undergo cysteine-dependent dimerization/oligomerization to be secreted. The cysteines engaged in this process have, in case of retention, previously been linked to ERp44 (Fig. 6), which is consistent with the concept that ERp44 traps incompletely assembled subunits. Although this concept is commonly referred to as "thiol-mediated retention," it is still unclear how these trapping interactions are formed. As ERp44 comprises a single-cysteine active site, the oxidizing equivalents to join two thiol groups would either have to be contributed by the binding partner (e.g., through an intramolecular disulfide or glutathionylation) or by ERp44 itself. The latter possibility could involve a disulfide-linked homodimer or a heterodimer composed of Ero1 $\alpha$ and ERp44. However, as detailed above, abundant Ero1 $\alpha-E R p 44$ complexes rather inhibit than promote "thiol-mediated retention."

\section{Conclusions and Perspectives}

Ero1 enzymes are an integral part in our understanding of redox maintenance in the ER. The two mammalian isoforms, which differ from the yeast enzyme in a number of aspects, fulfill similar roles in regulated disulfide production, but also display distinctive features. While recent work has begun to link these features to isoform-specific in vivo functions, there is certainly more room for discovery regarding the physiological roles of Ero1 $\alpha$ and Ero1 $\beta$. An eminent question for the future is how redundant these roles are in mammals. Given the viability of ERO1L/ERO1LB gene trap mice that still harbor detectable levels of the stress oxidase $\operatorname{Ero1} \beta$ (55), it is possible that Ero1 $\beta$ can largely substitute for Ero1 $\alpha$ deficiency. Motivated by the finding that cardiomyocytes show decreased excitability due to lowered calcium transients in ERO1L mutant mice (16), however, it will be important to more closely look at, for example, ER-stress-induced apoptosis or the secretion of ERp44 substrates in these mice. Moreover, the question whether ERO1L/ERO1LB knockout mice are also viable remains to be answered.

Since the reduction of $\mathrm{O}_{2}$ by Ero1 oxidases produces cytotoxic $\mathrm{H}_{2} \mathrm{O}_{2}$ in the ER, the function of ER-resident $\mathrm{H}_{2} \mathrm{O}_{2-}$ degrading peroxidases and their crosstalk with the PDI family are likely to be fundamental $(4,13)$. For instance, the activity of peroxiredoxin IV can produce disulfides and channel them into oxidative folding (56). As for Ero1-derived $\mathrm{H}_{2} \mathrm{O}_{2}$, it will be most interesting to carry out loss-of-function analyses with two other PDI peroxidases, GPx7 and GPx8, which physically interact with Ero1 $\alpha$ (40).

Many additional features of mammalian Ero1 enzymes still require further investigation. The functional significance of covalent Ero1 dimerization (18) that apparently involves Cys $^{166}$ in Erol $\alpha$ (29) is completely obscure. Likewise, the molecular basis of membrane association of Ero1 $\alpha$ is not known, and it remains to be clarified whether its ER retention/MAM localization is mostly mediated by interaction with the ER membrane $(14,42)$ or with ERp44 and other PDI family members (41). In addition, the conservation among metazoans of the N-terminal segment including two disulfides in Ero1 $\alpha$ (but not Ero1 $\beta$, Fig. 3) suggests some as yet enigmatic function for this stretch of amino acids. It will also be instrumental to molecularly describe the mode(s) of interaction between Ero1 $\alpha$ and ERp44 including the cysteine connectivities and subcellular localization of the complex (ER, ERGIC, or MAM) and compare it to the complex involving PDI (36). Since the catalytic Ero1 $\alpha-P D I$ interaction plays a critical role during the PDI-assisted translocation of the cholera toxin A1 subunit from the ER to the cytosol (38), it also remains to be explored, if endogenous ER-associated degradation substrates require this interplay. Further, the mechanism of redox-driven activation of Ero $1 \alpha / \beta$ remains to be elucidated, which - in conjunction with the aforementionedwill set the stage for more exciting Ero1 news in the future.

\section{Acknowledgments}

We thank Lars Ellgaard for comments on the article. Work in the authors' laboratory is funded by the Swiss National Science Foundation, the University of Basel, and the August Collin-Fonds. T.R. is a recipient of a Ph.D. fellowship by the Boehringer Ingelheim Fonds.

\section{References}

1. Adachi Y, Yamamoto K, Okada T, Yoshida H, Harada A, and Mori K. ATF6 is a transcription factor specializing in the regulation of quality control proteins in the endoplasmic reticulum. Cell Struct Funct 33: 75-89, 2008.

2. Anelli T, Alessio M, Bachi A, Bergamelli L, Bertoli G, Camerini S, Mezghrani A, Ruffato E, Simmen T, and Sitia R. Thiol-mediated protein retention in the endoplasmic reticulum: the role of ERp44. EMBO I 22: 5015-5022, 2003.

3. Anelli T, Bergamelli L, Margittai E, Rimessi A, Fagioli C, Malgaroli A, Pinton P, Ripamonti M, Rizzuto R, and Sitia R. Ero1 $\alpha$ regulates $\mathrm{Ca}^{2+}$ fluxes at the endoplasmic reticulummitochondria interface (MAM). Antioxid Redox Signal 16: 1077-1087, 2012.

4. Appenzeller-Herzog C. Glutathione- and non-glutathionebased oxidant control in the endoplasmic reticulum. I Cell Sci 124: 847-855, 2011.

5. Appenzeller-Herzog $C$ and Ellgaard L. The human PDI family: versatility packed into a single fold. Biochim Biophys Acta 1783: 535-548, 2008.

6. Appenzeller-Herzog C, Riemer J, Christensen B, Sorensen ES, and Ellgaard L. A novel disulphide switch mechanism in Erolalpha balances ER oxidation in human cells. EMBO J 27: 2977-2987, 2008.

7. Appenzeller-Herzog C, Riemer J, Zito E, Chin KT, Ron D, Spiess M, and Ellgaard L. Disulphide production by Ero1alpha-PDI relay is rapid and effectively regulated. EMBO I 29: 3318-3329, 2010.

8. Araki K and Inaba K. Structure, mechanism and evolution of Ero1 family enzymes. Antioxid Redox Signal 16: 790-799, 2012.

9. Araki $\mathrm{K}$ and Nagata K. Functional in vitro analysis of ERO1 and protein-disulfide isomerase (PDI) pathway. I Biol Chem 286: 32705-32712, 2011.

10. Baker KM, Chakravarthi S, Langton KP, Sheppard AM, Lu $\mathrm{H}$, and Bulleid NJ. Low reduction potential of Erolalpha regulatory disulphides ensures tight control of substrate oxidation. EMBO J 27: 2988-2997, 2008.

11. Benham AM, Cabibbo A, Fassio A, Bulleid N, Sitia R, and Braakman I. The CXXCXXC motif determines the folding, structure and stability of human Ero1-Lalpha. EMBO I 19: 4493-4502, 2000. 
12. Bertoli G, Simmen T, Anelli T, Molteni SN, Fesce R, and Sitia R. Two conserved cysteine triads in human Erolalpha cooperate for efficient disulfide bond formation in the endoplasmic reticulum. I Biol Chem 279: 30047-30052, 2004.

13. Bulleid NJ and Ellgaard L. Multiple ways to make disulfides. Trends Biochem Sci 36: 485-492, 2011.

14. Cabibbo A, Pagani M, Fabbri M, Rocchi M, Farmery MR, Bulleid NJ, and Sitia R. ERO1-L, a human protein that favors disulfide bond formation in the endoplasmic reticulum. I Biol Chem 275: 4827-4833, 2000.

15. Chambers JE, Tavender TJ, Oka OB, Warwood S, Knight D, and Bulleid NJ. The reduction potential of the active site disulfides of human protein disulfide isomerase limits oxidation of the enzyme by Erolalpha. I Biol Chem 285: 2920029207, 2010.

16. Chin KT, Kang G, Qu J, Gardner LB, Coetzee WA, Zito E, Fishman GI, and Ron D. The sarcoplasmic reticulum luminal thiol oxidase ERO1 regulates cardiomyocyte excitationcoupled calcium release and response to hemodynamic load. FASEB I 25: 2583-2591, 2011.

17. Decuypere JP, Monaco G, Bultynck G, Missiaen L, De Smedt $\mathrm{H}$, and Parys JB. The IP(3) receptor-mitochondria connection in apoptosis and autophagy. Biochim Biophys Acta 1813: 1003-1013, 2011.

18. Dias-Gunasekara S, Gubbens J, van Lith M, Dunne C, Williams JA, Kataky R, Scoones D, Lapthorn A, Bulleid NJ, and Benham AM. Tissue-specific expression and dimerization of the endoplasmic reticulum oxidoreductase Erolbeta. I Biol Chem 280: 33066-33075, 2005.

19. Fraldi A, Zito E, Annunziata F, Lombardi A, Cozzolino M, Monti M, Spampanato C, Ballabio A, Pucci P, Sitia R, and Cosma MP. Multistep, sequential control of the trafficking and function of the multiple sulfatase deficiency gene product, SUMF1 by PDI, ERGIC-53 and ERp44. $\underline{\text { Hum Mol }}$ Genet 17: 2610-2621, 2008.

20. Frand AR and Kaiser CA. The ERO1 gene of yeast is required for oxidation of protein dithiols in the endoplasmic reticulum. Mol Cell 1: 161-170, 1998.

21. Frand AR and Kaiser CA. Ero1p oxidizes protein disulfide isomerase in a pathway for disulfide bond formation in the endoplasmic reticulum. Mol Cell 4: 469-477, 1999.

22. Frand AR and Kaiser CA. Two pairs of conserved cysteines are required for the oxidative activity of Ero1p in protein disulfide bond formation in the endoplasmic reticulum. $\mathrm{Mol}$ Biol Cell 11: 2833-2843, 2000.

23. Gess B, Hofbauer KH, Wenger RH, Lohaus C, Meyer HE, and Kurtz A. The cellular oxygen tension regulates expression of the endoplasmic oxidoreductase ERO1-Lalpha. Eur J Biochem 270: 2228-2235, 2003.

24. Gilady SY, Bui M, Lynes EM, Benson MD, Watts R, Vance $\mathrm{JE}$, and Simmen T. Ero1alpha requires oxidizing and normoxic conditions to localize to the mitochondria-associated membrane (MAM). Cell Stress Chaperones 15: 619-629, 2010.

25. Gross E, Kastner DB, Kaiser CA, and Fass D. Structure of Ero1p, source of disulfide bonds for oxidative protein folding in the cell. Cell 117: 601-610, 2004.

26. Gross E, Sevier CS, Heldman N, Vitu E, Bentzur M, Kaiser CA, Thorpe C, and Fass D. Generating disulfides enzymatically: reaction products and electron acceptors of the endoplasmic reticulum thiol oxidase Ero1p. Proc Natl Acad Sci U S A 103: 299-304, 2006.

27. Heldman N, Vonshak O, Sevier CS, Vitu E, Mehlman T, and Fass D. Steps in reductive activation of the disulfidegenerating enzyme Ero1p. Protein Sci 19: 1863-1876, 2010.
28. Higo T, Hattori M, Nakamura T, Natsume T, Michikawa T, and Mikoshiba K. Subtype-specific and ER lumenal environmentdependent regulation of inositol 1,4,5-trisphosphate receptor type 1 by ERp44. Cell 120: 85-98, 2005.

29. Inaba K, Masui S, Iida H, Vavassori S, Sitia R, and Suzuki M. Crystal structures of human Erolalpha reveal the mechanisms of regulated and targeted oxidation of PDI. EMBO I 29: 3330-3343, 2010.

30. Jessop CE, Watkins RH, Simmons JJ, Tasab M, and Bulleid NJ. Protein disulphide isomerase family members show distinct substrate specificity: P5 is targeted to $\mathrm{BiP}$ client proteins. L Cell Sci 122: 4287-4295, 2009.

31. Khoo C, Yang J, Rajpal G, Wang Y, Liu J, Arvan P, and Stoffers DA. Endoplasmic reticulum oxidoreductase-1-Like \{beta\} (ERO1l\{beta\}) regulates susceptibility to endoplasmic reticulum stress and is induced by insulin flux in \{beta\}-cells. Endocrinology 152: 2599-2608, 2011.

32. Li G, Mongillo M, Chin KT, Harding H, Ron D, Marks AR, and Tabas I. Role of ERO1-alpha-mediated stimulation of inositol 1,4,5-triphosphate receptor activity in endoplasmic reticulum stress-induced apoptosis. L Cell Biol 186: 783-792, 2009.

33. Li G, Scull C, Ozcan L, and Tabas I. NADPH oxidase links endoplasmic reticulum stress, oxidative stress, and PKR activation to induce apoptosis. I Cell Biol 191: 1113-1125, 2010.

34. Marciniak SJ, Yun CY, Oyadomari S, Novoa I, Zhang Y, Jungreis R, Nagata $K$, Harding HP, and Ron D. CHOP induces death by promoting protein synthesis and oxidation in the stressed endoplasmic reticulum. Genes Dev 18: 30663077, 2004.

35. Mariappan M, Radhakrishnan K, Dierks T, Schmidt B, and von Figura K. ERp44 mediates a thiol-independent retention of formylglycine-generating enzyme in the endoplasmic reticulum. I Biol Chem 283: 6375-6383, 2008.

36. Masui S, Vavassori S, Fagioli C, Sitia R, and Inaba K. Molecular bases of cyclic and specific disulfide interchange between human ERO1\{alpha\} protein and protein-disulfide isomerase (PDI). L Biol Chem 286: 16261-16271, 2011.

37. May D, Itin A, Gal O, Kalinski H, Feinstein E, and Keshet E. Ero1-L alpha plays a key role in a HIF-1-mediated pathway to improve disulfide bond formation and VEGF secretion under hypoxia: implication for cancer. Oncogene 24: 10111020, 2005.

38. Moore P, Bernardi KM, and Tsai B. The Erolalpha-PDI redox cycle regulates retro-translocation of cholera toxin. Mol Biol Cell 21: 1305-1313, 2010.

39. Nadanaka S, Okada T, Yoshida H, and Mori K. Role of disulfide bridges formed in the luminal domain of ATF6 in sensing endoplasmic reticulum stress. Mol Cell Biol 27: 10271043, 2007.

40. Nguyen VD, Saaranen MJ, Karala AR, Lappi AK, Wang L, Raykhel IB, Alanen HI, Salo KE, Wang CC, and Ruddock LW. Two endoplasmic reticulum PDI peroxidases increase the efficiency of the use of peroxide during disulfide bond formation. I Mol Biol 406: 503-515, 2011.

41. Otsu M, Bertoli G, Fagioli C, Guerini-Rocco E, NeriniMolteni S, Ruffato E, and Sitia R. Dynamic retention of Erolalpha and Erolbeta in the endoplasmic reticulum by interactions with PDI and ERp44. Antioxid Redox Signal 8: 274-282, 2006.

42. Pagani M, Fabbri M, Benedetti C, Fassio A, Pilati S, Bulleid NJ, Cabibbo A, and Sitia R. Endoplasmic reticulum oxidoreductin 1-lbeta (ERO1-Lbeta), a human gene induced in the 
course of the unfolded protein response. I Biol Chem 275: 23685-23692, 2000.

43. Pagani M, Pilati S, Bertoli G, Valsasina B, and Sitia R. The Cterminal domain of yeast Ero1p mediates membrane localization and is essential for function. FEBS Lett 508: 117-120, 2001.

44. Pollard MG, Travers KJ, and Weissman JS. Ero1p: a novel and ubiquitous protein with an essential role in oxidative protein folding in the endoplasmic reticulum. Mol Cell 1: 171-182, 1998.

45. Qiang L, Wang H, and Farmer SR. Adiponectin secretion is regulated by SIRT1 and the endoplasmic reticulum oxidoreductase Ero1-L alpha. Mol Cell Biol 27: 4698-4707, 2007.

46. Schulman S, Wang B, Li W, and Rapoport TA. Vitamin K epoxide reductase prefers ER membrane-anchored thioredoxin-like redox partners. Proc Natl Acad Sci U S A 107: 15027-15032, 2010.

47. Sevier CS and Kaiser CA. Disulfide transfer between two conserved cysteine pairs imparts selectivity to protein oxidation by Ero1. Mol Biol Cell 17: 2256-2266, 2006.

48. Sevier CS, Qu H, Heldman N, Gross E, Fass D, and Kaiser CA. Modulation of cellular disulfide-bond formation and the ER redox environment by feedback regulation of Ero1. Cell 129: 333-344, 2007.

49. Tien AC, Rajan A, Schulze KL, Ryoo HD, Acar M, Steller H, and Bellen HJ. Ero1L, a thiol oxidase, is required for Notch signaling through cysteine bridge formation of the Lin12Notch repeats in Drosophila melanogaster. J Cell Biol 182: 11131125, 2008.

50. Tu BP, Ho-Schleyer SC, Travers KJ, and Weissman JS. Biochemical basis of oxidative protein folding in the endoplasmic reticulum. Science 290: 1571-1574, 2000.

51. Vitu E, Kim S, Sevier CS, Lutzky O, Heldman N, Bentzur M, Unger T, Yona M, Kaiser CA, and Fass D. Oxidative activity of yeast Ero1p on protein disulfide isomerase and related oxidoreductases of the endoplasmic reticulum. L Biol Chem 285: 18155-18165, 2010.

52. Wang L, Li SJ, Sidhu A, Zhu L, Liang Y, Freedman RB, and Wang CC. Reconstitution of human Ero1-Lalpha/proteindisulfide isomerase oxidative folding pathway in vitro. Position-dependent differences in role between the a and a' domains of protein-disulfide isomerase. L Biol Chem 284: 199_ 206, 2009.

53. Wang $\mathrm{L}$, Zhu L, and Wang CC. The endoplasmic reticulum sulfhydryl oxidase Erolbeta drives efficient oxidative protein folding with loose regulation. Biochem I 434: 113-121, 2011.
54. Wang ZV, Schraw TD, Kim JY, Khan T, Rajala MW, Follenzi $\mathrm{A}$, and Scherer PE. Secretion of the adipocyte-specific secretory protein adiponectin critically depends on thiolmediated protein retention. Mol Cell Biol 27: 3716-3731, 2007.

55. Zito E, Chin KT, Blais J, Harding HP, and Ron D. ERO1-beta, a pancreas-specific disulfide oxidase, promotes insulin biogenesis and glucose homeostasis. L Cell Biol 188: 821-832, 2010.

56. Zito E, Melo EP, Yang Y, Wahlander A, Neubert TA, and Ron D. Oxidative protein folding by an endoplasmic reticulumlocalized peroxiredoxin. Mol Cell 40: 787-797, 2010.

Address correspondence to: Dr. Christian Appenzeller-Herzog Division of Molecular and Systems Toxicology Department of Pharmaceutical Sciences University of Basel Klingelbergstrasse 50 CH-4056 Basel Switzerland

E-mail: christian.appenzeller@unibas.ch

Date of first submission to ARS Central, December 15, 2011; date of acceptance, December 18, 2011.

$\begin{array}{rl} & \text { Abbreviations Used } \\ \mathrm{CHOP}= & \mathrm{C} / \mathrm{EBP} \text { homologous protein } \\ \mathrm{ER}= & \text { endoplasmic reticulum } \\ \mathrm{ERGIC}= & \text { ER-Golgi intermediate compartment } \\ \mathrm{ErO} 1= & \text { endoplasmic oxidoreductin } 1 \\ \mathrm{FAD} & =\text { flavin adenine dinucleotide } \\ \mathrm{H}_{2} \mathrm{O}_{2}= & \text { hydrogen peroxide } \\ \mathrm{IgM} & =\text { immunoglobulin } \mathrm{M} \\ \mathrm{IP} & \mathrm{R}=\text { inositol } 1,4,5 \text {-trisphosphate receptor } \\ \mathrm{LPS} & =\text { lipopolysaccharide } \\ \mathrm{MAM} & =\text { mitochondria-associated membranes } \\ \mathrm{O}_{2}= & \text { molecular oxygen } \\ \mathrm{PDI}= & \text { protein disulfide isomerase } \\ \mathrm{PPAR} \gamma & =\text { peroxisome proliferator-activated } \\ & \text { receptor gamma } \\ \mathrm{ROS}= & \text { reactive oxygen species } \\ \mathrm{SUM} 1= & \text { sulfatase modifying factor } 1 \\ \mathrm{VEGF}= & \text { vascular endothelial growth factor } \\ & \end{array}$




\section{This article has been cited by:}

1. Benjamin A. Israel, Lingxi Jiang, Shawn A. Gannon, Colin Thorpe. 2014. Disulfide bond generation in mammalian blood serum: detection and purification of quiescin-sulfhydryl oxidase. Free Radical Biology and Medicine 69, 129-135. [CrossRef]

2. Thomas Ramming, Henning G. Hansen, Kazuhiro Nagata, Lars Ellgaard, Christian Appenzeller-Herzog. 2014. GPx8 peroxidase prevents leakage of $\mathrm{H} 2 \mathrm{O} 2$ from the endoplasmic reticulum. Free Radical Biology and Medicine . [CrossRef]

3. András Szarka, Tamás Lőrincz. 2013. The role of ascorbate in protein folding. Protoplasma . [CrossRef]

4. Tino Prell, Janin Lautenschläger, Julian Grosskreutz. 2013. Calcium-dependent protein folding in amyotrophic lateral sclerosis. Cell Calcium 54:2, 132-143. [CrossRef]

5. Shane Deegan, Svetlana Saveljeva, Adrienne M. Gorman, Afshin Samali. 2013. Stress-induced self-cannibalism: on the regulation of autophagy by endoplasmic reticulum stress. Cellular and Molecular Life Sciences 70:14, 2425-2441. [CrossRef]

6. A. M. Benham, M. van Lith, R. Sitia, I. Braakman. 2013. Ero1-PDI interactions, the response to redox flux and the implications for disulfide bond formation in the mammalian endoplasmic reticulum. Philosophical Transactions of the Royal Society B: Biological Sciences 368:1617, 20110403-20110403. [CrossRef]

7. Davide Montero, Christine Tachibana, Jakob Rahr Winther, Christian Appenzeller-Herzog. 2013. Intracellular glutathione pools are heterogeneously concentrated. Redox Biology 1:1, 508-513. [CrossRef]

8. Thomas Ramming, Christian Appenzeller-Herzog. 2013. Destroy and Exploit: Catalyzed Removal of Hydroperoxides from the Endoplasmic Reticulum. International Journal of Cell Biology 2013, 1-13. [CrossRef]

9. Lloyd W. Ruddock. 2012. Low-Molecular-Weight Oxidants Involved in Disulfide Bond Formation. Antioxidants \& Redox Signaling 16:10, 1129-1138. [Abstract] [Full Text HTML] [Full Text PDF] [Full Text PDF with Links]

10. Christian Appenzeller-Herzog, Michael N. Hall. 2012. Bidirectional crosstalk between endoplasmic reticulum stress and mTOR signaling. Trends in Cell Biology 22:5, 274-282. [CrossRef]

11. Taichi Kakihana, Kazuhiro Nagata, Roberto Sitia. 2012. Peroxides and Peroxidases in the Endoplasmic Reticulum: Integrating Redox Homeostasis and Oxidative Folding. Antioxidants \& Redox Signaling 16:8, 763-771. [Abstract] [Full Text HTML] [Full Text PDF] [Full Text PDF with Links]

12. Kazutaka Araki, Kenji Inaba. 2012. Structure, Mechanism, and Evolution of Ero1 Family Enzymes. Antioxidants \& Redox Signaling 16:8, 790-799. [Abstract] [Full Text HTML] [Full Text PDF] [Full Text PDF with Links] [Supplemental Material] 